\title{
An isomeric reaction benchmark set to test if the performance of state-of-the-art density functionals can be regarded as independent of the external potential $\uparrow$
}

Cite this: Phys. Chem. Chem. Phys., 2014, 16, 14559

\begin{abstract}
Tobias Schwabe
Some representative density functionals are assessed for isomerization reactions in which heteroatoms are systematically substituted with heavier members of the same element group. By this, it is investigated if the functional performance depends on the elements involved, i.e. on the external potential imposed by the atomic nuclei. Special emphasis is placed on reliable theoretical reference data and the attempt to minimize basis set effects. Both issues are challenging for molecules including heavy elements. The data suggest that no general bias can be identified for the functionals under investigation except for one case - M11-L. Nevertheless, large deviations from the reference data can be found for all functional approximations in some cases. The average error range for the nine functionals in this test is $17.6 \mathrm{kcal} \mathrm{mol}^{-1}$. These outliers depreciate the general reliability of density functional approximations.
\end{abstract}

Received 21st February 2014 Accepted 17th March 2014

DOI: $10.1039 / c 4 c p 00772 g$

www.rsc.org/pccp
These sets are either compiled to evaluate the broad applicability of a given functional ${ }^{10-13}$ or to evaluate its performance for a specific problem. ${ }^{14-16}$ Even mindless benchmarks have been advertised. ${ }^{17}$ While in the beginning the main goal was to reproduce experimental values nowadays the focus lies on the comparison with more sophisticated quantum chemical methods because this allows for a better judgment of the quality. In any case, the choice and compilation of a given benchmark set (can) bias the result.

Benchmark sets or subsets of them are naturally also involved in the parametrization of empirically trained functionals. Of course, the number of fitting points for the parametrization has to be limited and their choice is influenced by a number of considerations among which efficiency and the existence of reliable reference data are the most important. Another important aspect is the desired purpose of a functional. Sometimes they are trained for special problems although there is a general agreement that a good functional should perform equally well for as many problems as possible. Because of resource limitations during the development process it cannot be excluded that the composition of its parametrization set corrupts an empirically trained functional.

For example, molecules containing atoms of higher rows of the periodic table are less often part of parametrization or benchmark sets. The reason for this is just that molecules with heavy atoms are all but what they should be like. It takes more time to compute results for them because of the larger number of electrons, it is more difficult to obtain reliable reference results and the heavier elements are less frequently found in 
experimental chemistry and thereby are less of general interest. Although these molecules often show similar chemical behavior as their cousins containing lighter atoms of the same group the electronic structure is of course not identical. Especially the influence of inner (d-) shell electrons can be significant. This effect might be important not only for transition metal elements but also for main group elements. And of course, going to larger and larger atoms, relativistic effects would become more and more important and can have a significant influence depending on what molecular property is investigated. ${ }^{18}$ All this led to the present study where a set of representative functionals is tested for their dependency on the involved atoms. It is a fundamental statement of the Hohenberg-Kohn theorems that the exact functional is independent of the external potential which is imposed by the atomic nuclei. ${ }^{1,2}$ Here, it is tested if this requirement is fulfilled for a representative set of recent density functional approximations.

To assess the functionals a set of isomerization reactions has been compiled. Isomerization reactions have been found to be quite sensitive to the electronic structure method used for computing reaction energies. ${ }^{19-23}$ For each reaction the atom of interest is systematically exchanged with a heavier atom of the same group in the periodic table. To be independent of experimental data sophisticated reference data based on postHartree-Fock approaches are computed and serve to evaluate the performance of the functionals. Only main group elements are considered because for these elements the reference data are the most reliable. Relativistic effects have been investigated but for the problems at hand, i.e. the assessment of density functional approximations, they can be ignored as long as the treatment is consistent for the reference and evaluated methods. The chosen functional approximations represent state-of-the-art functionals which are known to be suitable for the problem at hand, especially because for the more recent ones, isomerization reactions are normally part of the training set in the development process. The assessment is presented as follows. First, computational details are given, followed by a detailed presentation of the test set. Next, the computed reference data are discussed before the actual evaluation of the functionals is given. Finally, some concluding remarks are drawn.

\section{Computational details}

All geometries are optimized with PBE0-D3 ${ }^{24,25} /$ def2-TZVPP. ${ }^{26}$ Energies in the SCF and geometry optimization steps have been converged to $10^{-7}$ a.u. The density integration has been carried out with the multiple grid $\mathrm{m} 4 .^{27}$ Based on these geometries, single point energies have been computed with SCS-MP2, ${ }^{28}$ B2GP-PLYP-D3, ${ }^{29}$ PBE0-D3, B3LYP-D3, ${ }^{30,31}$ PW6B95-D3, ${ }^{32}$ M06-2X, ${ }^{33,34}$ oTPSS-D3, ${ }^{35} \omega \mathrm{B} 97 \mathrm{X}-\mathrm{D},{ }^{36}$ M11, ${ }^{37}$ and M11-L ${ }^{38}$ all in combination with a cc-p(wC)V5Z basis set. The latter labels a basis set where the cc-pV5Z basis set ${ }^{39,40}$ is used for all atoms except for those of the second and third rows (here, $\mathrm{Si}, \mathrm{P}, \mathrm{S}, \mathrm{Cl}, \mathrm{Ge}$, As, Se, and $\mathrm{Br}$ ) for which the cc-pwCV5Z basis set ${ }^{41,42}$ is applied.
The empirical dispersion correction is Grimme's 2010 version (D3) ${ }^{43}$ as implemented in the applied program packages.

Finally, single point energies have also been computed with $\operatorname{CCSD}(\mathrm{T})^{44} / \mathrm{cc}-\mathrm{p}(\mathrm{wC}) \mathrm{VTZ}$ and SCS-MP2/cc-p(wC)VTZ to obtain energy corrections for higher correlation effects. For some reactions, even more sophisticated computations based on the cc-p(wC)VQZ basis set have been carried out (see ESI $\dagger$ ).

For all wavefunction based correlation methods, the resolution-of-the-identity approximation in combination with the corresponding auxiliary basis sets has been used for the correlation treatment. ${ }^{45-49}$ Further, a partial frozen core treatment has been applied. That is, only valence electrons are considered except for the heavier elements (here, Si, P, S, Cl, Ge, As, Se, and $\mathrm{Br}$ ) where the $n-1$ shell is also taken into account. Following a recent suggestion, ${ }^{50}$ this scheme has also been applied to the double-hybrid functional. To estimate the effect of coreelectron correlation, $\operatorname{CCSD}(\mathrm{T}) / \mathrm{cc}-\mathrm{pVTZ}$ results with a full frozen core approximation have also been computed.

Single point energies for PW6B95-D3 are obtained using ORCA, version $2.9,{ }^{51}$ for $\omega$ B97X-D using version 3.0. Both versions make use of the Libint2 Library. ${ }^{52}$ For an estimation of relativistic effects, PW6B95-D3/def2-QZVPP ${ }^{26}$ results with and without the ZORA approximation ${ }^{53}$ (using a corresponding decontracted basis set ${ }^{54}$ ) are also computed. The standard integration grid is always used.

Computations for M06-2X are carried out using NWChem 6.0, M11 and M11-L with version 6.3. ${ }^{55}$ To avoid problems with the meta(hybrid) functionals, the integration grid $x$ fine, the largest standard grid, was chosen.

All other computations are done using a locally modified version of TURBOMOLE $6.4 .^{56}$

\section{Composition of the test set}

The test set consists of several isomerization reactions which are listed in Fig. 1. They are arranged by the element group of the heteroatom. In all reactions the chemical environment for the heteroatom changes considerably, i.e. the atom is not just part of a (conserved) functional group, such that error compensation is less likely with respect to the treatment of that atom. Further, the first seven reactions for elements of groups 14,15 , and 16 are chosen to represent similar isomerizations which allow for some comparison between groups. Because of their monovalency, the set of reactions for group 17 elements contains different and less isomerizations. Reactions 10 for group 15 and 16 is only considered for second and third row atoms because they include molecules which are sometimes labeled hypervalent. Regardless whether these molecules feature a special kind of bonding the electronic structure can be seen as an extreme case because of large polarization effects at the heteroatom nucleus. Therefore, these molecules are taken into account. Finally, for carbon reaction 3 is trivial because it yields the identical molecule and reaction 2 is identical to reaction 1 . The latter is also true for reactions 8 and 9 in the case of carbon. These reactions are not considered therefore. 


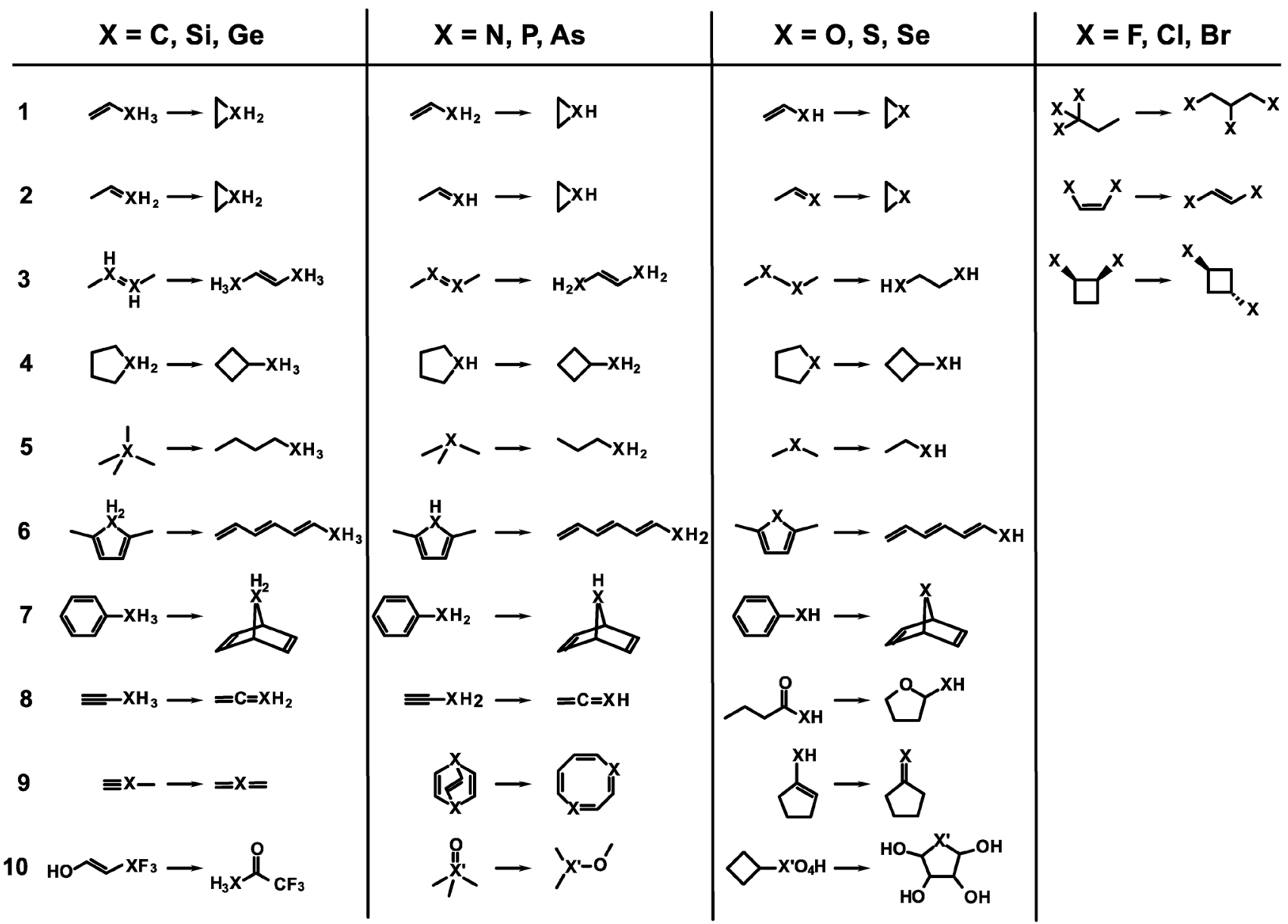

Fig. 1 Scheme for all isomerization reactions included in the test set. Primes on generic heavy atoms $\mathrm{X}$ indicate that onlythe 2 nd and 3 rd row elements are considered.

In total, the test set consists of 94 isomerization reactions. For the ease of reference, reactions are labeled by the element of interest and the reaction entry, like for example Se.07, which is the seventh reaction for group 16 with selenium as a heteroatom.

\section{Results and discussion}

\subsection{Reference data}

To obtain reliable reference data, the following strategy has been chosen. First, SCS-MP2/cc-p(wC)V5Z results are obtained. These results are then corrected for higher correlation effects based on $\operatorname{CCSD}(\mathrm{T})$ computations to yield the final $\Delta E$ (ref):

$$
\begin{aligned}
\Delta E(\mathrm{ref})= & \Delta E(\mathrm{SCS}-\mathrm{MP} 2 / \mathrm{cc}-\mathrm{p}(\mathrm{wC}) \mathrm{V} 5 \mathrm{Z}) \\
& +(\Delta E(\mathrm{CCSD}(\mathrm{T}) / \mathrm{cc}-\mathrm{p}(\mathrm{wC}) \mathrm{VTZ}) \\
& -\Delta E(\mathrm{SCS}-\mathrm{MP} 2 / \mathrm{cc}-\mathrm{p}(\mathrm{wC}) \mathrm{VTZ}))
\end{aligned}
$$

The reference data for the isomerization reactions under consideration are collected in Table 1 . They span the range from 0.0 to $153.7 \mathrm{kcal} \mathrm{mol}^{-1}$ (absolute values) which indicates the challenging character of the test set because very different changes in the electronic structure occur for the individual reactions.
On average, the correction for higher correlation effects amounts to $0.6 \mathrm{kcal} \mathrm{mol}^{-1}$ with a minimum of -4.6 and a maximum of $2.4 \mathrm{kcal} \mathrm{mol}^{-1}$ (see ESI $\dagger$ for SCS-MP2 results). The difference between the $\operatorname{CCSD}(\mathrm{T})(\mathrm{FC}) / \mathrm{cc}-\mathrm{pVTZ}$ and $\operatorname{CCSD}(\mathrm{T})$ (partial FC)/cc-p(wC)VTZ treatments is $1.0 \mathrm{kcal} \mathrm{mol}^{-1}$ on average (only considering the second and third row results). The largest deviation in that case is $-18.4 \mathrm{kcal} \mathrm{mol}^{-1}$ for reaction $\mathrm{S} .10 \mathrm{but}$ note that already the $\mathrm{HF}$ results differ by $-17.0 \mathrm{kcal} \mathrm{mol}^{-1}$. Obviously, additional core-polarization functions are needed already at the SCF level to treat the electronic structure in a balanced way. On the other hand, for reaction Ge.03, the difference is only $0.4 \mathrm{kcal} \mathrm{mol}^{-1}$ on the SCF level, but -5.0 between the two $\operatorname{CCSD}(\mathrm{T})$ treatments.

To show that this way of approximating higher correlation effects is not very sensitive to the choice of the lower correlation method, the corrected SCS-MP2 results are compared to the corrected MP2 results (see ESI $\dagger$ ). On average, both sets deviate by less than $0.1 \mathrm{kcal} \mathrm{mol}^{-1}$ in absolute numbers with a maximum deviation of $0.3 \mathrm{kcal} \mathrm{mol}^{-1}$.

It is known that even the quintuple- $\zeta$ basis set might not yield results at the complete basis set limit although only relative energies are considered. To estimate this effect SCS-MP2 results extrapolated to the complete basis set limit have been computed 
Table 1 Reference isomerization energies for the test set based on SCSMP2/cc-p $(w C) V 5 Z$ computations with $\operatorname{CCSD}(T)$ corrections for higher correlation contributions (see text). All values are in $\mathrm{kcal} \mathrm{mol}^{-1}$

\begin{tabular}{|c|c|c|c|c|}
\hline Ret & Group 14 & Group 15 & Group 16 & Group 17 \\
\hline \multicolumn{5}{|c|}{ First row } \\
\hline 1 & 5.9 & 16.6 & 15.8 & 26.9 \\
\hline 2 & - & 17.9 & 25.2 & 1.0 \\
\hline 3 & - & -20.9 & -63.7 & -2.7 \\
\hline 4 & 17.7 & 12.3 & 9.9 & \\
\hline 5 & 3.8 & -12.0 & -12.4 & \\
\hline 6 & 18.9 & 32.0 & 27.4 & \\
\hline 7 & 45.1 & 57.1 & 58.8 & \\
\hline 8 & 1.3 & -14.2 & 19.6 & \\
\hline 9 & - & -31.8 & -11.5 & \\
\hline 10 & -9.1 & - & - & \\
\hline \multicolumn{5}{|c|}{ Second row } \\
\hline 1 & 6.4 & -4.9 & -2.6 & -3.3 \\
\hline 2 & -10.1 & -1.7 & -0.9 & 0.6 \\
\hline 3 & -3.9 & 23.0 & 6.6 & -1.6 \\
\hline 4 & 28.2 & 26.1 & 19.8 & \\
\hline 5 & 33.4 & 10.8 & -1.2 & \\
\hline 6 & 21.5 & 6.6 & 35.5 & \\
\hline 7 & 28.9 & 35.3 & 47.1 & \\
\hline 8 & 22.0 & -3.9 & 11.9 & \\
\hline 9 & -4.5 & 17.9 & -3.0 & \\
\hline 10 & 153.7 & 33.2 & -15.2 & \\
\hline \multicolumn{5}{|c|}{ Third row } \\
\hline 1 & 8.0 & -7.9 & -6.8 & -6.3 \\
\hline 2 & -7.4 & -5.7 & -5.1 & 0.1 \\
\hline 3 & 14.2 & 38.7 & 16.0 & -1.6 \\
\hline 4 & 26.7 & 27.2 & 20.8 & \\
\hline 5 & 24.6 & 9.8 & 0.0 & \\
\hline 6 & 19.7 & -4.8 & 32.8 & \\
\hline 7 & 30.7 & 33.0 & 43.4 & \\
\hline 8 & 19.1 & -4.2 & 10.7 & \\
\hline 9 & -2.3 & -3.1 & -2.9 & \\
\hline 10 & 93.5 & -2.9 & -68.9 & \\
\hline
\end{tabular}

(see ESI $\dagger$ for details). On average, the results change by $0.2 \mathrm{kcal} \mathrm{mol}^{-1}$ for absolute deviations with a maximum of $1.0 \mathrm{kcal} \mathrm{mol}^{-1}$ for reaction Ge.10. In addition, for the first three reactions with all heteroatoms under consideration (34 data points in total) $\mathrm{CCSD}(\mathrm{T}) / \mathrm{cc}-\mathrm{p}(\mathrm{wC}) \mathrm{VQZ}$ results are also obtained and are extrapolated to the complete basis set limit (see ESI $\dagger$ for details). This allows us to test the correction for higher correlation effects.

To compute results for heavier elements which can be compared to experimental data relativistic effects have to be taken into account. To estimate these effects for the present test set, corrections from PW6B95-D3/def-QZVPP with and without the ZORA approximation have been computed. These corrections are small, $0.4 \mathrm{kcal} \mathrm{mol}^{-1}$ on average for absolute values and even only 0.5 when only 2nd and 3rd row results are considered. Nevertheless, for single cases the correction can be up to $7.6 \mathrm{kcal} \mathrm{mol}^{-1}$ (for Se.10) even for closed shell systems (see ESI $\dagger$ for all results). This effect cannot be neglected when chemical accuracy is aimed for. On the other hand, for the present study only relative performances between electronic structure methods are of interest. Therefore, the effect can safely be ignored as long as every method is treated on equal footing. Even if relativistic corrections would be computed based on the different approaches, the actual variation between methods should be below $1.0 \mathrm{kcal} \mathrm{mol}^{-1}$.
Based on these numbers, a conservative estimate is a maximum deviation of $1.0 \mathrm{kcal} \mathrm{mol}^{-1}$ from the reference results and the true $\operatorname{CCSD}(\mathrm{T})$ results at the quintuple- $\zeta$ level presumably even for an all electron treatment (although that would require a basis set which is trained for such a treatment). For those reaction energies for which $\operatorname{CCSD}(\mathrm{T})$ results have been computed with a larger basis set (see ESI $\dagger$ ), estimating $\operatorname{CCSD}(\mathrm{T})$ values or actually compute them yields virtually the same results. The mean absolute deviation is $0.1 \mathrm{kcal} \mathrm{mol}^{-1}$ with a maximum deviation of $0.4 \mathrm{kcal} \mathrm{mol}^{-1}$. A direct judgment of the results' quality with respect to the true electronic structure is not possible. Based on the present data, no estimate can be given for what the effect of even higher correlation terms would be. But as none of the calculations shows a significant multi-reference character and given the general belief that such higher correlation terms are only significant for results beyond the chemical accuracy, it might be safe to assume no larger effect than an additional deviation of $1.0 \mathrm{kcal} \mathrm{mol}^{-1}$. Finally, the basis set incompleteness could add another $1.0 \mathrm{kcal} \mathrm{mol}^{-1}$ but is most often less severe. In principle, complete basis set limit results could be estimated for all methods yet such an approach is less established for the various DFT methods at hand. Further, different kind of basis sets might be needed for consistent treatment. Therefore, all results are compared for the same basis set. Again, the aforementioned error estimations should be regarded as conservative ones for challenging cases. For most of the values within the test set, the reliability should be higher especially because often the applied approximations have opposing effects and cancel to some extent. Therefore, each result deviating more than $3 \mathrm{kcal} \mathrm{mol}^{-1}$ form the reference result is regarded as an outlier in the following discussion.

\subsection{Functional assessment}

Based on the reference data, nine representative functionals are assessed. For comparison, the results regarding SCS-MP2, which is also often recommended for such computations, are also given. Key values for the assessment are listed in Table 2 . Detailed results can be found in the ESI. $\dagger$

A standard criterion for evaluating quantum mechanical methods is the mean absolute deviation (MAD). SCS-MP2 shows

Table 2 Key values for functional performance. Given are the mean deviation $(\bar{x})$, the mean absolute deviation $(|\bar{x}|)$, the median of the absolute deviation $(|\tilde{x}|)$, the maximum $\left(x_{\max }\right)$ and minimum deviations $\left(x_{\min }\right)$, and the number of outliers (\#). All energy values are in $\mathrm{kcal} \mathrm{mol}^{-1}$

\begin{tabular}{lrrrrrr}
\hline Functional & \multicolumn{1}{l}{$\bar{x}$} & $|\overline{\mathrm{x}}|$ & $|\tilde{x}|$ & $x_{\max }$ & \multicolumn{1}{c}{$x_{\min }$} & $\#$ \\
\hline SCS-MP2(FC) & 0.4 & 1.0 & 0.6 & 4.6 & -3.7 & 6 \\
B2GP-PLYP-D3(FC) & 0.5 & 1.3 & 0.4 & 7.8 & -4.6 & 11 \\
B3LYP-D3 & 0.0 & 3.6 & 2.1 & 12.6 & -11.7 & 34 \\
PBE0-D3 & -0.5 & 2.1 & 2.0 & 6.0 & -7.1 & 22 \\
M06-2X & -0.2 & 1.9 & 1.1 & 8.7 & -8.6 & 21 \\
PW6B95-D3 & -0.6 & 1.8 & 1.6 & 5.7 & -5.5 & 14 \\
$\omega$ B97X-D & -0.4 & 2.0 & 1.5 & 5.3 & -8.2 & 21 \\
M11 & -0.3 & 2.4 & 1.5 & 6.3 & -14.6 & 34 \\
M11-L & -0.6 & 3.7 & 2.8 & 13.9 & -19.2 & 44 \\
OTPSS-D3 & -0.2 & 2.7 & 2.1 & 13.2 & -8.5 & 31
\end{tabular}


the lowest MAD of $1.0 \mathrm{kcal} \mathrm{mol}^{-1}$, followed by B2GP-PLYP-D3

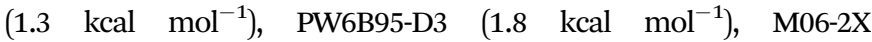
$\left(1.9 \mathrm{kcal} \mathrm{mol}^{-1}\right), \quad \omega$ B97X-D $\left(2.0 \quad \mathrm{kcal}^{-1} \mathrm{~mol}^{-1}\right), \quad$ PBE0-D3 (2.1 kcal mol $\left.{ }^{-1}\right)$, M11 (2.4 kcal mol $\left.{ }^{-1}\right)$, oTPSS-D3 (2.7 kcal mol $\left.{ }^{-1}\right)$, B3LYP-D3 (3.5 kcal mol ${ }^{-1}$ ) and M11-L (3.7 kcal mol $\left.{ }^{-1}\right)$.

These numbers hide to some extent the individual performance for a given set entry. To give an impression of that one can inspect the range of deviations for each method. From the smallest to the largest, the order is: SCS-MP2 (8.3 kcal mol $\left.{ }^{-1}\right)$, PW6B95-D3 (11.2 kcal mol ${ }^{-1}$ ), B2GP-PLYP-D3 (12.4 kcal mol ${ }^{-1}$ ), PBE0-D3 (13.1 kcal mol ${ }^{-1}$ ), $\omega$ B97X-D (13.5 kcal mol $\left.{ }^{-1}\right)$, M06-2X (17.3 kcal mol ${ }^{-1}$ ), M11 (20.9 kcal mol ${ }^{-1}$ ), oTPSS-D3 (21.7 kcal mol ${ }^{-1}$ ), B3LYP-D3 (24.3 kcal mol ${ }^{-1}$ ), and M11-L (33.1 kcal mol ${ }^{-1}$ ).

Further, MAD values are sensitive to outliers. An alternative criterion which can be used to assess methods is the median of the absolute deviations. Again, this changes the ranking of the methods under investigation: B2GP-PLYP-D3 (0.4 kcal mol $\left.{ }^{-1}\right)$, SCS-MP2 (0.6 kcal mol $\left.{ }^{-1}\right)$, M06-2X (1.1 kcal mol $\left.{ }^{-1}\right)$, M11 and $\omega$ B97X-D (1.5 kcal mol ${ }^{-1}$ ), PW6B95-D3 (1.6 kcal mol $\left.{ }^{-1}\right)$, PBE0D3 (1.7 kcal mol ${ }^{-1}$ ), B3LYP-D3 (2.0 kcal mol ${ }^{-1}$ ), oTPSS-D3 (2.1 $\mathrm{kcal} \mathrm{mol}^{-1}$ ) and M11-L (2.8 kcal mol $\left.{ }^{-1}\right)$.

As a final way to compare all methods, the distribution of deviations for each methods is given in Fig. 2. SCS-MP2 and B2GP-PLYP-D3 show a sharp peak around a deviation of $0 \mathrm{kcal} \mathrm{mol}{ }^{-1}$. For the latter a distinct group of outliers around $7 \mathrm{kcal} \mathrm{mol}^{-1}$ can be identified. For M11-L and B3LYP-D3, a completely different distribution, namely a flat and broad one, is obtained. The remaining functionals lie somewhere in between these two patterns. Interestingly, PW6B95-D3 is the only functional that shows it maximum not around $0 \mathrm{kcal} \mathrm{mol}^{-1}$, but around $-2 \mathrm{kcal} \mathrm{mol}^{-1}$. These distributions can also be complemented by the number of outliers (deviations larger than $3 \mathrm{kcal} \mathrm{mol}^{-1}$ ) which is 6 for SCS-MP2, 11 for B2GP-PLYP-D3, 14 for PW6B95-D3, 21 for M06-2X and $\omega$ B97X-D, 22 for PBE0-D3, 31 for oTPSS-D3, 34 for M11 and B3LYP-D3, and 44 for M11-L.

Some of the functionals are supplemented with an empirical dispersion correction. For the present benchmark set, which contains relatively small molecules, the effect of the correction is negligible. For completeness, results without the correction are given in the ESI. $\dagger$ For some rare cases the effect is as large as $2.0 \mathrm{kcal} \mathrm{mol}^{-1}$, but most often it is well below $0.5 \mathrm{kcal} \mathrm{mol}^{-1}$. And there is no consistent improvement due to the correction so that the overall effect cancels for the statistics. Actual values for some cases with the largest isomerization energy change are listed in Table 3 . As it is generally recommended to apply a

Table 3 Overview of reactions where the largest effect of the dispersion correction is found. Given are the dispersion correction contributions to the deviation from the reference data. A negative sign means improvement. All values are in $\mathrm{kcal} \mathrm{mol}^{-1}$

\begin{tabular}{lrrrrr}
\hline & \multicolumn{2}{l}{ Reaction label } & \\
\cline { 2 - 6 } Functional & C.05 & C.06 & P.09 & S.10 & Ge.05 \\
\hline B2GP-PLYP-D3 & -0.6 & -0.2 & 0.7 & -0.8 & 0.8 \\
B3LYP-D3 & -1.6 & -0.7 & 1.4 & -1.9 & 1.4 \\
PBE0-D3 & -1.0 & 0.5 & 0.8 & -1.0 & 0.6 \\
PW6B95-D3 & -0.7 & -0.2 & 0.7 & -0.9 & 0.8 \\
WB97X-D & -0.6 & 0.7 & -0.5 & -0.3 & 0.1 \\
oTPSS-D3 & -1.7 & -1.0 & 1.6 & -2.0 & 1.5
\end{tabular}

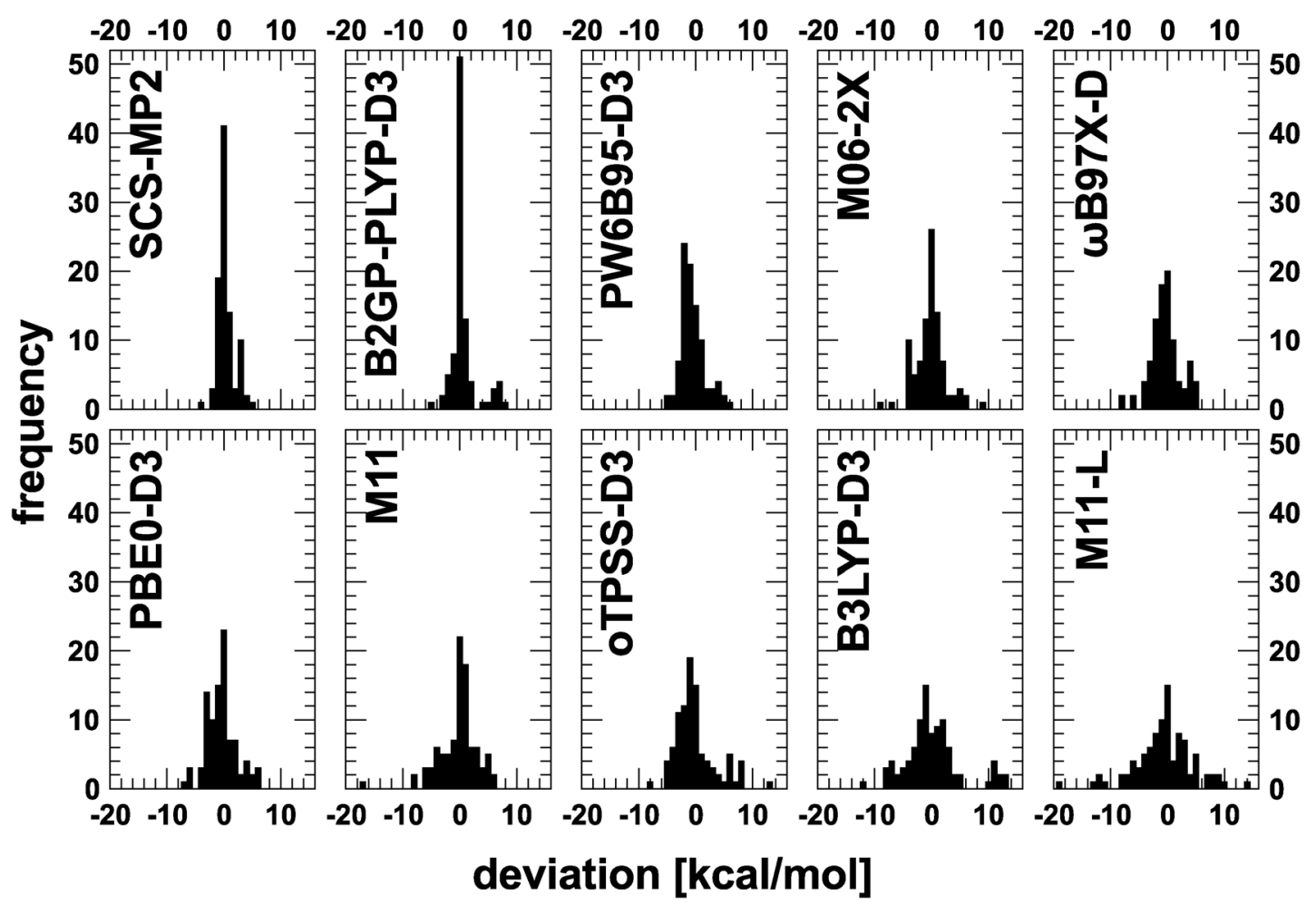

Fig. 2 Error distributions for several functionals with respect to reference data. 
Table 4 Key values for functional performance grouped by rows of the heteroatoms. Given are the mean deviation $(\bar{x})$, the mean absolute deviation $(|\bar{x}|)$, and the median of the absolute deviation $(|\tilde{x}|)$. All values are in $\mathrm{kcal} \mathrm{mol}^{-1}$

\begin{tabular}{|c|c|c|c|c|c|c|c|c|c|}
\hline \multirow[b]{2}{*}{ Functional } & \multicolumn{3}{|c|}{1 st row } & \multicolumn{3}{|c|}{ 2nd row } & \multicolumn{3}{|c|}{ 3rd row } \\
\hline & $\bar{x}$ & $|\bar{x}|$ & $|\tilde{x}|$ & $\bar{x}$ & $|\bar{x}|$ & $|\tilde{x}|$ & $\bar{x}$ & $|\bar{x}|$ & $|\tilde{x}|$ \\
\hline SCS-MP2(FC) & 0.2 & 0.7 & 0.6 & 0.4 & 1.2 & 0.6 & 0.5 & 1.0 & 0.6 \\
\hline B2GP-PLYP-D3(FC) & 0.6 & 1.3 & 0.4 & 0.6 & 1.3 & 0.7 & 0.4 & 1.2 & 0.3 \\
\hline B3LYP-D3 & 0.4 & 3.5 & 1.7 & -0.1 & 3.8 & 2.2 & -0.3 & 3.4 & 1.8 \\
\hline PBE0-D3 & -0.1 & 1.9 & 1.8 & -0.5 & 2.3 & 1.8 & -0.9 & 2.1 & 2.1 \\
\hline M06-2X & 0.0 & 1.7 & 1.1 & -0.6 & 1.7 & 0.7 & -0.1 & 2.2 & 1.8 \\
\hline PW6B95-D3 & -0.2 & 1.7 & 1.3 & -0.6 & 1.9 & 1.6 & -0.9 & 1.9 & 1.8 \\
\hline$\omega$ B97X-D & 0.2 & 1.9 & 1.5 & -0.5 & 2.0 & 1.4 & -0.8 & 2.0 & 1.5 \\
\hline M11 & 0.2 & 1.8 & 0.9 & -0.2 & 2.4 & 2.1 & -0.8 & 2.8 & 1.5 \\
\hline M11-L & 0.4 & 1.8 & 0.7 & -0.2 & 4.1 & 2.9 & -1.9 & 4.9 & 3.2 \\
\hline oTPSS-D3 & -0.6 & 2.7 & 2.3 & 0.0 & 2.6 & 2.1 & 0.0 & 2.7 & 1.5 \\
\hline
\end{tabular}

dispersion correction, only these results are discussed in the following.

Now, the statistical key values are grouped by the row the heteroatom belongs to. The results are shown in Table 4. While also of empirical nature to some extent, SCS-MP2 is less likely to show a bias because of the rather small number of only two very general parameters needed to define the approach. ${ }^{28}$ Comparing the results of SCS-MP2 against the DFT methods, most of the latter show a similar performance dependency on the periodic table row of the heteroatom - the mean absolute deviations as well as the medians of the absolute deviations can be considered to be quite stable for increasing the atom size within a group for almost all functionals under consideration. The worst case is found for M11-L for which the median increases by $2.5 \mathrm{kcal} \mathrm{mol}^{-1}$ from first to third row results ( $3.1 \mathrm{kcal} \mathrm{mol}^{-1}$ for the MAD). It is also the only functional where a continuous relationship between error statistics and the element row can be identified.

To put these numbers into context, the results regarding reactions 1 to 7 for groups 14,15 , and 16 are also compared against each other. For each group of the periodic table, these reactions describe similar isomerizations. Because of this similarity, the functional performance should be very consistent for a given reaction when compared with the heteroatoms of the same row. Therefore, the results for the group-wise comparison should reveal the variation in the performance which has to be expected for each functional when it is applied to related problems. The statistical key values are given in Table 5 .

The variations in statistics which can be found are in the same range as in Table 4. Slight fluctuations can be related to the limited number of test cases and to the fact that, of course, the group-wise test sets are not completely identical. The same noise in functional statistics can also be assumed for the deviations for different rows of the periodic table. Therefore, any bias due to systematic dependency effects which are smaller than the general functional performance variation cannot be resolved. Besides very consistent results for almost all functionals, M11-L, stands out because the error statistics for a group-wise analysis deviate less than they do for a row-wise analysis. For the former, the maximum deviation of medians of the absolute error is only $1.5 \mathrm{kcal} \mathrm{mol}^{-1}$.
Table 5 Key values for functional performance according to the group of the heteroatoms. Given are the mean deviation $(\bar{x})$, the mean absolute deviation $(|\bar{x}|)$, and the median of the absolute deviation $(|\tilde{x}|)$. All values are in $\mathrm{kcal} \mathrm{mol}^{-1}$

\begin{tabular}{|c|c|c|c|c|c|c|c|c|c|}
\hline \multirow[b]{2}{*}{ Functional } & \multicolumn{3}{|c|}{ Group 14} & \multicolumn{3}{|c|}{ Group 15} & \multicolumn{3}{|c|}{ Group 16} \\
\hline & $\bar{x}$ & $|\bar{x}|$ & $|\tilde{x}|$ & $\bar{x}$ & $|\bar{x}|$ & $|\tilde{x}|$ & $\bar{x}$ & $|\bar{x}|$ & $|\tilde{x}|$ \\
\hline SCS-MP2(FC) & 0.3 & 1.2 & 0.7 & 0.5 & 1.0 & 0.8 & 0.4 & 1.0 & 0.5 \\
\hline B2GP-PLYP-D3(FC) & 0.3 & 0.9 & 0.3 & 0.3 & 1.5 & 0.5 & 1.2 & 1.2 & 0.5 \\
\hline B3LYP-D3 & -0.5 & 2.4 & 1.4 & -0.8 & 4.0 & 2.8 & 1.7 & 3.7 & 2.1 \\
\hline PBE0-D3 & -1.3 & 2.0 & 2.1 & -0.7 & 2.6 & 2.3 & 0.4 & 1.9 & 1.8 \\
\hline M06-2X & -0.2 & 1.5 & 0.6 & -0.4 & 2.3 & 1.5 & -0.1 & 1.9 & 1.5 \\
\hline PW6B95-D3 & -1.3 & 1.6 & 1.4 & -1.0 & 2.2 & 1.8 & 0.5 & 1.8 & 1.8 \\
\hline$\omega \mathrm{B} 97 \mathrm{X}-\mathrm{D}$ & -0.4 & 2.1 & 2.4 & -0.3 & 2.3 & 2.1 & 0.4 & 1.9 & 1.4 \\
\hline M11 & -0.3 & 2.3 & 1.5 & 0.3 & 2.5 & 2.1 & 0.3 & 2.4 & 1.8 \\
\hline M11-L & -1.4 & 4.4 & 3.9 & -0.4 & 3.4 & 2.3 & 0.7 & 2.9 & 2.1 \\
\hline oTPSS-D3 & -0.6 & 2.6 & 2.3 & -0.5 & 2.5 & 2.1 & 0.4 & 2.7 & 1.9 \\
\hline
\end{tabular}

According to the arguments given before, such a performance indicates a dependency on the row of heteroatom involved in the reaction. To further analyse the performance of M11-L, for each reaction the relative change in the deviation with respect to data from first row atoms is plotted for the second and third row data sets. A dependency on the type of heteroatom should result in a systematic change for going to the heavier elements. The changes are plotted in Fig. 3 along with results regarding M11 and oTPSS-D3.

The plot reveals that the M11-L performance shows systematic trends for almost all reactions considered here. The amount of deviation from reference data is increasing for the heavier heteroatoms. Such a pattern cannot be found for the other two functionals and also for none of the other functionals in the present study (see ESI $\dagger$ ). Like M11-L, oTPSS is a meta-GGA functional containing a set of fitted parameters but with a very different functional form. On the other hand, the functional forms of M11 and M11-L are very similar, though the former belongs to the class of range-separated hybrid functionals. M11-L also incorporates the idea of range-separation but in a local fashion, termed dual-range (density functional) exchange. This is a unique characteristic and few studies with this approach of functional design can be found in the literature. Therefore, it is not possible to conclude from the present data if this could be a general problem or is just a fitting bias in the design of the functional. The comparison to the related functionals M11-L and oTPSS might hint of the latter. In any case, this deserves further analysis and is beyond the scope of the present paper.

\section{Conclusion and summary}

Nine representative functionals have been assessed for a set of isomerization reactions for which the key heteroatom has been successively substituted by a heavier member of the element group. This investigation was designed to reveal any bias which could be found for a given functional which is often trained only on a subset of elements during the development. The results are compared against very sophisticated reference data which have been computed at the SCS-MP2/cc-p(wC)V5Z level of theory corrected by $\operatorname{CCSD}(\mathrm{T}) / \mathrm{cc}-\mathrm{p}(\mathrm{wC}) \mathrm{VTZ}$ values for higher correlation effects. 


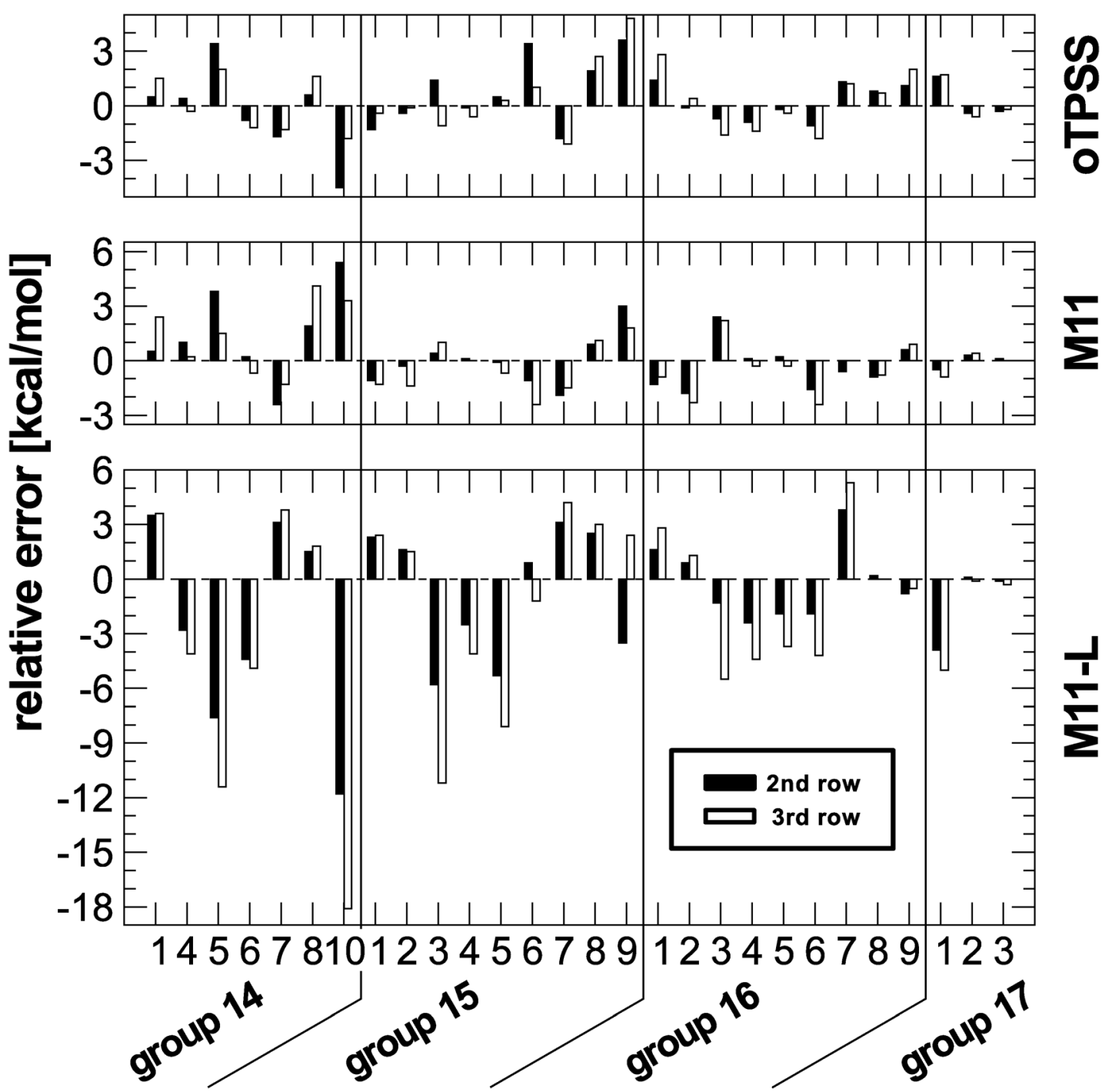

Fig. 3 Relative change in deviations with respect to first row results for a given isomerization reaction set.

Based on the data presented in this study no general bias can be identified. Most functionals show a stable performance downwards the periodic table and vary only to such an amount which has to be expected for similar but not identical chemical problems. Of course, this has to be expected for an exact density functional which only depends on the electron density and not on the external potential. But this cannot be guaranteed for an approximate density functional - at least when it is based on (some) empiricism. In one case, M11-L, some dependency on the heteroatom involved could be revealed. While this might be seen as a violation of the Hohenberg-Kohn theorems, one should remember that care has always to be taken when applying trained methods outside their proposed purposes. For the 1st row atoms, M11-L is among the best functionals in this test set and also has demonstrated its good performance before. $^{38}$ The present test set can help to reparameterize the functional and might eliminate the bias while maintaining the general performance if no general flaw is found for local range-separated functionals.
That no general bias can be found for the given test set once more justifies the application of good functional approximations for very diverse tasks in quantum chemistry. Nevertheless, only a small though hopefully representative set of DFAs has been tested. Further, only isomerization energies have been considered. Although this is a very sensitive test for electronic structure methods, future studies should also take other properties into account to check how severe any bias for a specific group of elements is for a given DFA. For now, the present benchmark set can already serve as a first test to identify the possible problems in DFAs not considered so far or for new developments.

Yet, showing a constant performance across the periodic table is worth nothing if that means constantly poor results. None of the functionals can beat SCS-MP2 for this test set in terms of the error distribution and general reliability. At the same time it has a similar computational cost as the double-hybrid B2GP-PLYP-D3. The latter shows the next best performance. Even more interesting, if its outliers could be eliminated B2GP-PLYP-D3 should outperform SCS-MP2. 
That such an improved double-hybrid functional could be found might be seen from the fact that there are a few reactions for which all of the tested functionals show systematically deviations larger than $3 \mathrm{kcal} \mathrm{mol}^{-1}$. Most of these cases for which all functionals perform poorly can be related to reaction $\mathrm{X} .07$ in which the aromatic system in the phenyl ring is destroyed. The aromatic system is always too stable. This indicates that the self-interaction error common to practically all approximate density functionals deteriorates the results.

Finding solutions to remedy the problem of self-interaction while maintaining the superior cost-performance ratio with respect to sophisticated wave function methods persists to be the great challenge in density functional development. Ideas like the rangeseparated double-hybrids, ${ }^{57,58}$ the MCY approach, ${ }^{59}$ or even higherorder approaches like the random-phase-approximation ${ }^{60}$ or the $G W$-method ${ }^{61}$ might be solutions. But these methods still have to prove that they do not loose the generality of common density functionals which are not only successful for main group chemistry but for problems all across the table. At least the present study emphasizes again the fact that functional performance is transferable to related chemistry.

\section{References}

1 R. G. Parr and W. Yang, Density-Functional Theory of Atoms and Molecules, Oxford University Press, Oxford, 1989.

2 W. Koch and C. Holthausen, A Chemist's Guide to Density Functional Theory, Wiley-VCH, Weinheim, New York, Chichester, Brisbane, Singapore, Toronto, 2000.

3 P. Mori-Sánchez, A. J. Cohen and W. Yang, J. Chem. Phys., 2006, 125, 201102.

4 A. J. Cohen, P. Mori-Sáchez and W. Yang, Chem. Rev., 2012, 112, 289-320.

5 X. Zheng, M. Liu, E. R. Johnson, J. Contreras-García and W. Yang, J. Chem. Phys., 2012, 137, 214106.

6 K. E. Riley, M. Pitok, P. Jureka and P. Hobza, Chem. Rev., 2010, 110, 5023-5063.

7 S. Grimme, Wiley Interdiscip. Rev.: Comput. Mol. Sci., 2011, 1, 211-228.

8 T. M. Henderson and G. E. Scuseria, Mol. Phys., 2010, 108, 2511-2517.

9 E. R. Johnson, J. Contreras-García and W. Yang, J. Chem. Theory Comput., 2012, 8, 2676-2681.

10 L. A. Curtiss, P. C. Redfern and K. Raghavachari, J. Chem. Phys., 2006, 125, 201102.

11 K. P. Jensen, Inorg. Chem., 2008, 47, 10357-10365.

12 L. Goerigk and S. Grimme, Phys. Chem. Chem. Phys., 2011, 13, 6670-6688.

13 Y. Zhao and D. G. Truhlar, J. Chem. Theory Comput., 2011, 7, 669-676.

14 T. Schwabe and S. Grimme, J. Phys. Chem. Lett., 2010, 1, 201-204.

15 M. Srebro, N. Govind, W. A. de Jong and J. Autschbach, J. Phys. Chem. A, 2011, 115, 10930-10943.
16 S. S. Leang, F. Zahariev and M. S. Gordon, J. Chem. Phys., 2012, 136, 104101.

17 M. Korth and S. Grimme, J. Chem. Theory Comput., 2009, 5, 993-1003.

18 P. Pyykkö, Chem. Rev., 2012, 112, 371-384.

19 S. Grimme, M. Steinmetz and M. Korth, J. Org. Chem., 2007, 72, 2118-2126.

20 R. Huenerbein, B. Schirmer, J. Moellmann and S. Grimme, Phys. Chem. Chem. Phys., 2010, 12, 6940-6948.

21 S. Luo, Y. Zhao and D. G. Truhlar, Phys. Chem. Chem. Phys., 2011, 13, 13683-13689.

22 T. Schwabe, R. Huenerbein and S. Grimme, Synlett, 2010, 1431-1441.

23 T. Schwabe, J. Comput. Chem., 2012, 33, 2067-2072.

24 J. P. Perdew, K. Burke and M. Enzerhof, Phys. Rev. Lett., 1996, 77, 3865.

25 C. Adamo and V. J. Barone, J. Chem. Phys., 1999, 110, 6158.

26 F. Weigend and R. Ahlrichs, Phys. Chem. Chem. Phys., 2005, 7, 3297-3305.

27 O. Treutler and R. Ahlrichs, J. Chem. Phys., 1995, 102, 346.

28 S. Grimme, J. Chem. Phys., 2003, 118, 9095.

29 A. Karton, A. Tarnopolsky, J.-F. Lamere, G. C. Schatz and J. M. L. Martin, J. Phys. Chem. A, 2008, 112, 12868.

30 A. D. Becke, J. Chem. Phys., 1993, 98, 5648.

31 P. J. Stephens, F. J. Devlin, C. F. Chabalowski and M. J. Frisch, J. Phys. Chem., 1994, 98, 11623.

32 Y. Zhao and D. G. Truhlar, J. Phys. Chem. A, 2005, 109, 5656-5667.

33 Y. Zhao and D. G. Truhlar, Theor. Chem. Acc., 2008, 120, 215-241.

34 Y. Zhao and D. G. Truhlar, Theor. Chem. Acc., 2008, 119, 525.

35 L. Goerigk and S. Grimme, J. Chem. Theory Comput., 2010, 6, 107-126.

36 J.-D. Chai and M. Head-Gordon, Phys. Chem. Chem. Phys., 2008, 10, 16615-16620.

37 R. Peverati and D. G. Truhlar, J. Chem. Phys. Lett., 2011, 2, 2810-2817.

38 R. Peverati and D. G. Truhlar, J. Chem. Phys. Lett., 2011, 3, 117-124.

39 J. T. H. Dunning, J. Chem. Phys., 1989, 90, 1007-1023.

40 D. E. Woon and T. H. Dunning, J. Chem. Phys., 1993, 98, 1358-1371.

41 K. A. Peterson and J. T. H. Dunning, J. Chem. Phys., 2002, 117, 10548-10560.

42 N. J. DeYonker, K. A. Peterson and A. K. Wilson, J. Phys. Chem. A, 2007, 111, 11383-11393.

43 S. Grimme, J. Antony, S. Ehrlich and H. Krieg, J. Chem. Phys., 2010, 132, 154104.

44 T. Helgaker, P. Jörgensen and J. Olsen, Molecular ElectronicStructure Theory, J. Wiley, New York, 2000.

45 F. Weigend and M. Häser, Theor. Chem. Acc., 1997, 97, 331-340.

46 F. Weigend, M. Häser, H. Patzelt and R. Ahlrichs, Chem. Phys. Lett., 1998, 294, 143-152.

47 F. Weigend, A. Köhn and C. Hättig, J. Chem. Phys., 2002, 116, 3175-3183. 
48 C. Hättig, Phys. Chem. Chem. Phys., 2005, 7, 59-66.

49 A. Hellweg, C. Hättig, S. Höfener and W. Klopper, Theor. Chem. Acc., 2007, 117, 587-597.

50 T. Schwabe, J. Phys. Chem. A, 2013, 117, 2879-2883.

51 F. Neese, Wiley Interdiscip. Rev.: Comput. Mol. Sci., 2012, 2, 73-78.

52 E. Valeev, 2011, available from http://libint.valeyev.net.

53 C. van Wüllen, J. Chem. Phys., 1998, 109, 392-399.

54 D. A. Pantazis, X. Y. Chen, C. R. Landis and F. Neese, J. Chem. Theory Comput., 2008, 5, 908-919.

55 M. Valiev, E. J. Bylaska, N. Govind, K. Kowalski, T. P. Straatsma, H. J. J. van Dam, D. Wang, J. Nieplocha, E. Apra, T. L. Windus and W. A. de Jong, Comput. Phys. Commun., 2010, 181, 1477.
56 TURBOMOLE GmbH, 2012, TURBOMOLE V6.4, developed by University of Karlsruhe and Forschungszentrum Karlsruhe GmbH, 1989-2007, TURBOMOLE GmbH, since 2007; available from http:/www.turbomole.com.

57 J. H. Ángyán, I. C. Gerber, A. Savin and J. Toulouse, Phys. Rev. A: At., Mol., Opt. Phys., 2005, 72, 012510.

58 J.-D. Chai and M. Head-Gordon, J. Chem. Phys., 2009, 131, 174105.

59 P. Mori-Sánchez, A. J. Cohen and W. Yang, J. Chem. Phys., 2006, 124, 091102.

60 H. Eshuis, J. E. Bates and F. Furche, Theor. Chem. Acc., 2012, 131, 1084-1101.

61 M. J. van Setten, F. Weigend and F. Evers, J. Chem. Theory Comput., 2013, 9, 232-246. 